\section{Original Article}

Korean J Transplant 2020;34:257-264 https://doi.org/10.4285/kjt.20.0048
Received October 8, 2020

Revised November 9, 2020

Accepted November 9, 2020

Corresponding author: Kyu Ha Huh Department of Surgery, Yonsei University College of Medicine, 50-1 Yonsei-ro, Seodaemun-gu, Seoul 03722, Korea Tel: +82-2-2228-2138

Fax: +82-2-313-8289

E-mail: khhuh@yuhs.ac

(c) The Korean Society for Transplantation This is an Open Access article distributed under the terms of the Creative Commons Attribution Non-Commercial License (http://creativecommons.org/licenses/ by-nc/4.0/) which permits unrestricted non-commercial use, distribution, and reproduction in any medium, provided the original work is properly cited.

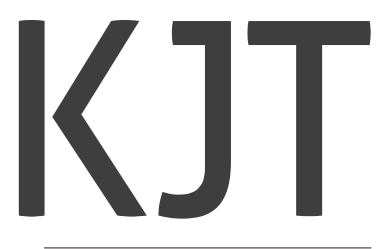

pISSN 2671-8790

elSSN 2671-8804

\title{
The feasibility of organ transplantation during the COVID-19 outbreak: experiences from South Korea
}

\author{
Juhan Lee, Eun Jin Kim, Kyong Ihn, Jae Geun Lee, Dong Jin Joo, Myoung Soo Kim, \\ Soon II Kim, Yu Seun Kim, Kyu Ha Huh
}

Department of Surgery, Yonsei University College of Medicine, Seoul, Korea

\begin{abstract}
Background: The coronavirus disease 2019 (COVID-19) has forced healthcare systems to reduce transplant activities in order to preserve resources and minimize the risk of nosocomial transmission. Although transplantation societies around the world have proposed interim recommendations, little is known about the safety of transplant surgery under pandemic conditions and how transplant medicine should move forward after the peak of the pandemic.

Methods: We describe our experiences regarding the continuation of living and deceased donor transplantation under infection control measures during the COVID-19 outbreak in South Korea. We reviewed consecutive liver and kidney transplantations at Severance Hospital and analyzed national transplantation activities in South Korea.

Results: Transplantation activities with living and deceased donors remained stable during the COVID-19 outbreak compared to the same period in 2019. We performed 94 transplantations (58 kidney, 35 liver, and 1 simultaneous liver-kidney) during the COVID-19 outbreak. Twenty-five patients underwent desensitization therapy prior to transplant (nine ABO-incompatible kidney, eight human leukocyte antigen-incompatible kidney, and eight $\mathrm{ABO}$-incompatible liver). No transplant recipients in our center contracted COVID-19. In South Korea, national transplant activities with living and deceased donors remained stable in 2020 compared to 2019.

Conclusions: Organ transplantation during pandemics appears to be feasible with appropriate infection prevention measures.
\end{abstract}

Keywords: COVID-19; Organ transplantation; Immunosuppression; Safety

\section{INTRODUCTION}

The coronavirus disease 2019 (COVID-19) spread rapidly around the world, causing the World Health Organization to declare a global pandemic on March 11, 2020. As of April 30, 2020, COVID-19 was confirmed in over 100 countries, causing more than $3,000,000$ confirmed cases and 223,193 deaths globally [1]. Although the case fatality rate is low in the general population, it is higher among patients with advanced age or underlying comorbidities [2]. In the absence of a vaccine or effective treatment, questions arise on the risk of COVID-19 to patients with end-stage organ disease that are awaiting transplant surgery $[3,4]$.

Organ transplantation saves thousands of lives; however, transplant recipients, because of immunosuppression and underlying medical conditions, might be at higher risk 


\section{HIGHLIGHTS}

- We describe our experiences regarding the continuation of living and deceased donor transplantation during the initial phase of the coronavirus disease 2019 (COVID-19) outbreak.

-We also analyzed national transplant activities using Korean Network for Organ Sharing (KONOS) database.

- Transplantation activities with living and deceased donors remained stable during the COVID-19 outbreak and no transplant recipients in our center contracted COVID-19.

- Our early experiences suggest that organ transplantation is feasible during the COVID-19 pandemic under appropriate infection prevention measures and extensive testing.

of contracting COVID-19 and developing severe illness [5]. As the pandemic worsens around the world, procurements of organs from deceased donors have decreased, and transplantations from living donors have been postponed to preserve medical resources for COVID-19 and to avoid nosocomial transmission $[6,7]$.

During the COVID-19 pandemic, decision-making about whether to proceed with organ transplantation is challenging. Although transplantation societies have proposed interim recommendations, little is known about the safety and feasibility of organ transplantation under enhanced infection prevention measures during the COVID-19 outbreak [5]. We report on the measures we have taken to reduce the transmission of COVID-19 and our experiences with living donor and deceased donor transplants in the initial phase of the COVID-19 pandemic in South Korea.

\section{METHODS}

All study procedures were conducted in accordance with the Declaration of Helsinki and were approved by the Institutional Review Board of Severance Hospital (IRB No. 2020-0500-001). Requirement for informed consent was waived by the Institutional Review Board because of the study's retrospective design. All living donations were voluntary, and all donors underwent evaluation by transplant surgeons, hepatologists, nephrologists, and clinical psychologists. All deceased donors were brain dead. No donor organs were obtained from executed prisoners or other institutionalized persons.

\section{Data Collection}

In this retrospective study, we reviewed consecutive liver transplantations and kidney transplantations performed at the Severance Hospital Transplant Center from January 20, 2020 to April 30, 2020. We also analyzed transplantations performed at our hospital during the same period in 2019 (January 20 to April 30). Severance Hospital is a 2,400bed, university-affiliated tertiary care hospital in Seoul, located about $240 \mathrm{~km}$ (150 miles) from the epicenter of the COVID-19 pandemic in South Korea (Daegu and North Gyeongsang province). The Severance Hospital Transplant Center, one of the largest Korean centers for organ transplantation, performs approximately 180 kidney transplants and 120 liver transplants annually. We reviewed electronic medical records, laboratory findings, and radiological examinations of all recipients and donors. We also analyzed data from the Korean Network for Organ Sharing (KONOS) database, which contains national data on organ donations and transplantations $[8,9]$.

\section{Infection Prevention Measures}

We established a makeshift dedicated triage for patients with fever or respiratory symptoms to prevent COVID-19 exposure to patients and healthcare providers. All patients and visitors to the hospital were screened using a standard questionnaire and thermal imaging cameras. We adopted universal face masking policy for healthcare workers in all clinical settings. In addition, healthcare workers who come into direct or indirect contact with patients with confirmed COVID-19 underwent testing for the disease subsequently.

Preoperative patients were screened for COVID-19 using real time reverse transcription polymerase chain reaction (RT-PCR) of nasopharyngeal swabs in accordance with institutional guidelines. As the situation changed, testing criteria for COVID-19 in preoperative patients were modified accordingly (Fig. 1). Initially, we adopted preoperative COVID-19 testing for patients with respiratory symptoms (February 4; 16 confirmed cases in South Korea), followed by testing of patients who had recently traveled to or stayed in high risk countries (February 6; 23 confirmed cases in South Korea), patients from the South Korean epicenter region of Daegu and North Gyeongsang province (February 24; 763 confirmed cases in South Korea), and immunocompromised patients (February 27; 1,595 


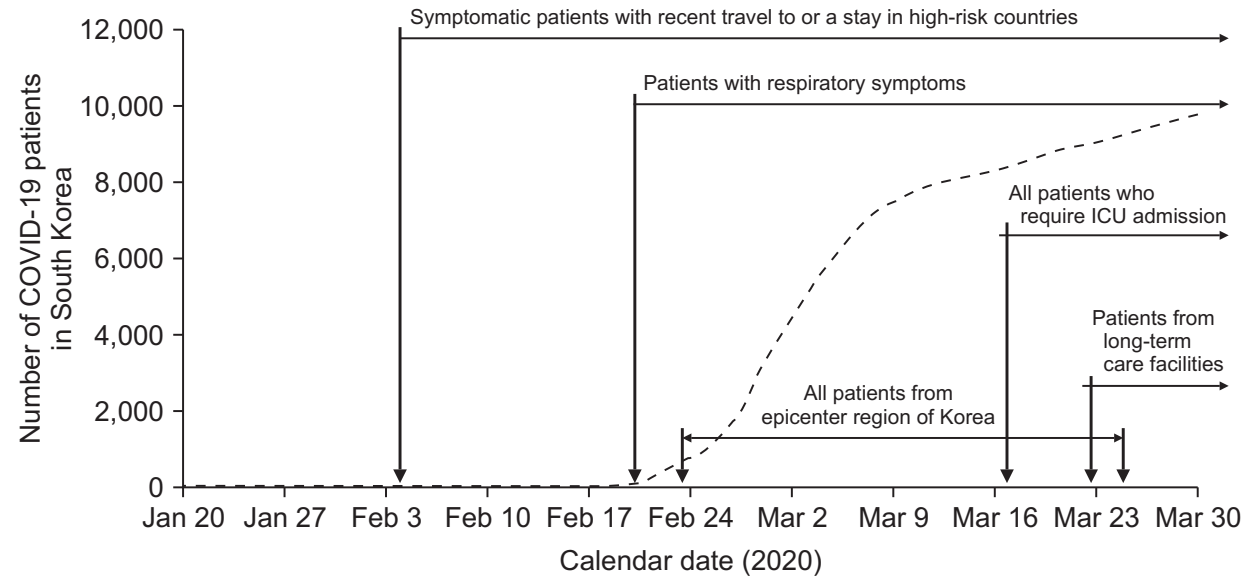

Fig. 1. Preoperative coronavirus disease 2019 (COVID-19) testing criteria during the outbreak. ICU, intensive care unit. confirmed cases in South Korea). Currently, all donors and recipients are screened for COVID-19 regardless of symptoms in accordance with the Korean Society for Transplantation recommendation (released on March 13, 2020) [10].

In addition, surgeries for patients from the epicenter region were scheduled after all other surgeries were performed in a negative pressure operating theater. After surgery, patients from the epicenter recovered from anesthesia in the operating theater instead of the postanesthesia care unit and were then sent directly back to the ward to minimize contact with other patients.

\section{Immunosuppressive Regimen}

Immunosuppression was performed as described previously [11]. Most patients that received living donor transplants received induction therapy with basiliximab $(20 \mathrm{mg}$ on days 0 and 4 posttransplant). We used anti-thymocyte globulin (ATG) for induction in human leukocyte antigen (HLA)-incompatible kidney transplantation and deceased donor kidney transplantation $(1.5 \mathrm{mg} / \mathrm{kg}$ per day for 4 days). The maintenance regimen consisted of tacrolimus, corticosteroid, and mycophenolate mofetil (MMF). Initial tacrolimus was administered orally at $0.1 \mathrm{mg} / \mathrm{kg}$ twice daily. The dose of tacrolimus was adjusted to maintain serum levels at $5-10 \mathrm{ng} / \mathrm{mL}$ during the first postoperative month and at $5-7 \mathrm{ng} / \mathrm{mL}$ thereafter. MMF was started at $1.0-1.5$ $\mathrm{g} /$ day and subsequently adjusted to minimize adverse events such as leukopenia or gastrointestinal trouble.

All ABO-incompatible transplantations and HLA-incompatible kidney transplantations were performed according to the desensitization protocol, as previously described $[11,12]$. Rituximab was administered within 2 weeks before transplantation. Plasmapheresis was performed until the target antibody titer was achieved (immunoglobulin $\mathrm{G}$ titer $\leq 1: 32$ in ABO-incompatible transplantation, conversion of a positive crossmatch to negative).

\section{Prophylaxis Protocol}

All patients received trimethoprim-sulfamethoxazole as Pneumocystis jirovecii pneumonia prophylaxis for a minimum of 3 months. Fungal prophylaxis consisted of $4 \mathrm{~mL}$ oral nystatin administered four times daily for 12 months. Prophylactic ganciclovir or valganciclovir for cytomegalovirus (CMV) prophylaxis was used in high risk patients (donor seropositive, recipient seronegative, or use of ATG induction). A preemptive approach for prevention of CMV infection was used in other patients.

\section{Statistical Analysis}

Demographic information was summarized using frequency counts (percentage) or medians (interquartile range), depending on the data type. The chi-square test with Fisher's exact test was used to compare categorical variables. The Kruskal-Wallis test was used to compare continuous variables. The analysis was performed using IBM SPSS ver. 25.0 (IBM Corp., Armonk, NY, USA). The P-values $<0.05$ were considered significant.

\section{RESULTS}

\section{Organ Transplant Activities}

During the COVID-19 outbreak, transplant activities remained stable compared to the same period in the previous year. In 2020 at Severance Hospital, 94 organ 
Table 1. Baseline characteristics of LT patients

\begin{tabular}{lccr}
\hline \multicolumn{1}{c}{ Variable } & LT in $2019(\mathrm{n}=36)$ & $\mathrm{LT}$ in $2020\left(\mathrm{n}=36^{\mathrm{a})}\right)$ & P-value \\
\hline Age & $53.0(38.8-61.8)$ & $54.5(45.3-62.8)$ & 0.813 \\
Elderly $(\geq 60 \mathrm{yr})$ & $12(33.3)$ & $11(30.6)$ & 0.800 \\
Female sex & $14(38.9)$ & $12(33.3)$ & 0.624 \\
Original disease & & & 0.439 \\
Hepatitis B virus & $12(33.3)$ & $14(38.9)$ & \\
Hepatitis C virus & 0 & $3(8.3)$ & \\
Alcoholic liver disease & $12(33.3)$ & $10(27.8)$ & \\
Biliary disease & $5(13.9)$ & $2(5.6)$ & \\
Others & $7(19.4)$ & $7(19.4)$ & \\
Hepatocellular carcinoma & $10(27.8)$ & $12(33.3)$ & 0.609 \\
Pretransplant loco- & $9 / 10(90.0)$ & $10 / 12(83.3)$ & \\
$\quad$ regional treatment & & & \\
MELD score & $14.0(10.8-28.3)$ & $18.0(11.8-32.3)$ & 0.988 \\
Diabetes & $13(33.3)$ & $13(33.3)$ & $>0.999$ \\
Deceased donor & $7(19.4)$ & $10(27.8)$ & 0.405 \\
ABO-incompatible LT & $6(16.7)$ & $8(22.2)$ & 0.551
\end{tabular}

Values are presented as median (interquartile range) or number (\%). LT, liver transplantation; MELD, model for end-stage liver disease.

a) One simultaneous liver-kidney transplant case was classified to LT.

transplantations were performed (58 kidney, 35 liver, and 1 simultaneous liver-kidney) compared to 84 in 2019 (48 kidney, and 36 liver). The proportion of living-donor transplantations was not significantly different between the 2 years.

\section{Baseline Characteristics}

The baseline characteristics of the liver transplant recipients are shown in Table 1. There were no significant differences between the COVID-19 period and the same time period in the previous year in terms of patient age, sex, and model for end-stage liver score. In both periods, alcoholic liver disease and hepatitis B were the two most common etiologies. A total of 22 patients had hepatocellular carcinoma at the time of liver transplant, and 19 underwent pretransplant locoregional treatment. The proportion of ABO-incompatible liver transplants was not significantly different between two periods $(22.2 \%$ in 2020 vs. $16.7 \%$ in 2019).

A total of 58 patients underwent kidney transplantation during the COVID-19 outbreak. The median age of those patients was 51 years, and $48.3 \%$ were female. Forty-two $(72.4 \%)$ of the patients that received kidney transplants during the COVID-19 outbreak had living donors, and 17 $(29.3 \%)$ underwent desensitization treatment prior to
Table 2. Baseline characteristics of KT patients

\begin{tabular}{|c|c|c|c|}
\hline Variable & KT in $2019(n=48)$ & KT in $2020(n=58)$ & P-value \\
\hline Age & $52.5(41.3-58.0)$ & $51.0(45.3-60.0)$ & 0.845 \\
\hline Elderly ( $\geq 60 \mathrm{yr}$ ) & $10(20.8)$ & $17(29.3)$ & 0.319 \\
\hline Female sex & $18(37.5)$ & $28(48.3)$ & 0.265 \\
\hline Number of HLA mismatch & $3.0(2.0-4.0)$ & $2.5(1.0-4.0)$ & 0.281 \\
\hline Retransplant & $5(10.4)$ & $5(8.6)$ & 0.753 \\
\hline Diabetes & $16(33.3)$ & $21(36.2)$ & 0.757 \\
\hline Deceased donor & $9(18.8)$ & $16(27.6)$ & 0.286 \\
\hline Desensitization & $16(33.3)$ & $17(29.3)$ & 0.656 \\
\hline ABO-incompatible KT & 10 & 9 & \\
\hline HLA-incompatible $\mathrm{KT}^{\mathrm{a}}$ ) & 6 & 8 & \\
\hline Induction & & & 0.124 \\
\hline Basiliximab & $35(72.9)$ & $34(58.6)$ & \\
\hline Anti-thymocyte globulin & $13(27.1)$ & $24(41.4)$ & \\
\hline \multicolumn{4}{|c|}{$\begin{array}{l}\text { Values are presented as median (interquartile range) or number (\%). } \\
\text { KT, kidney transplantation; HLA, human leukocyte antigen. } \\
\text { a) Five patient underwent both HLA- and ABO-incompatible kidney trans } \\
\text { plant was classified to HLA-incompatible kidney transplant. }\end{array}$} \\
\hline
\end{tabular}

transplantation (9 ABO-incompatible and 8 HLA-incompatible). The baseline characteristics of the kidney transplant patients are summarized in Table 2. There were no significant differences between the COVID-19 period and the same time period in the previous year in terms of age, sex, and diabetes. The use of ATG induction was more common in 2020 than in 2019, but the difference was not statistically significant $(41.4 \%$ in 2020 vs. $27.1 \%$ in 2019 , $\mathrm{P}=0.124)$.

\section{COVID-19 Test}

As of April 30, more than 620,000 individuals were tested for COVID-19 in South Korea (population of 51 million). A total of 7,550 patients underwent COVID-19 testing by real-time RT-PCR according to institutional guidelines at Severance Hospital. Among the patients undergoing transplantation, 50 recipients and 46 donors underwent COVID-19 testing prior to transplantation. During the study period, 909 healthcare workers were tested, and all were negative for COVID-19.

\section{Postoperative Courses}

The postoperative courses are summarized in Table 3. No incidence of COVID-19 infection occurred during the study period. All liver transplant recipients received care in the intensive care unit (ICU) after transplantation. The median length of stay in the ICU and duration of ventilator care 
Table 3. Postoperative courses after transplantation

\begin{tabular}{llcl}
\hline \multicolumn{1}{c}{ Variable } & 2019 & 2020 & P-value \\
\hline Liver transplant & $(\mathrm{n}=36)$ & $(\mathrm{n}=36)$ & \\
Length of ICU stay after transplant & $3(2-7)$ & $3(2-4)$ & 0.948 \\
Length of ventilator care & $2(1-5)$ & $1(1-3)$ & 0.227 \\
Continuous renal replacement therapy & $8(22.2)$ & $10(27.8)$ & 0.586 \\
Kidney transplant & $(\mathrm{n}=48)$ & $(\mathrm{n}=58)$ & \\
ICU care after transplant & $2(4.2)$ & $1(1.7)$ & 0.589 \\
Delayed graft function & $3(6.3)$ & $7(12.1)$ & 0.343 \\
\hline
\end{tabular}

Values are presented as median (interquartile range) or number (\%).

ICU, intensive care unit.

after liver transplantation were not significantly different between the COVID-19 period and the same time period in the previous year. In addition, the proportion of patients who received postoperative continuous renal replacement therapy was similar between the two time periods. Postoperative courses in kidney transplant recipients were not significantly different between the two time periods.

\section{Organ Donation and Transplant Activities in Korea}

We compared the organ donation and transplant activities from January 2020 to April 2020 with those during the same time period in the previous year (Table 4). According to the KONOS database, a total of 162 deceased donors were used in 2020 compared to 152 in 2019 [8]. Transplant activities were stable between the two time periods for both living donor and deceased donor transplantations [9]. In 2020, 1,409 organ transplantations were performed (543 liver, 774 kidney, 53 heart, and 39 lung) compared to 1,389 in 2019 (515 liver, 757 kidney, 66 heart, and 51 lung). The proportion of living donor transplantations was not significantly different between the two time periods.

\section{DISCUSSION}

Since the first imported case of COVID-19 was documented on January 20, 2020, South Korea has carried out extensive virus testing and contact tracing [13-15]. In cooperation with efforts at the national level, our institution put stringent measures into place to prevent COVID-19 transmission. As the situation continues to change rapidly, infection control measures for patients undergoing organ transplantation have been modified accordingly. Thanks to those efforts, we were able to maintain both living donor
Table 4. Overall organ transplant activities in South Korea

\begin{tabular}{cccc}
\hline Variable & Jan-Apr 2019 & Jan-Apr 2020 & $\begin{array}{c}\text { Change of } \\
\text { transplant } \\
\text { activities (\%) }\end{array}$ \\
\hline Deceased donor & 152 & 162 & +6.6 \\
Elderly ( $\geq 60$ yr) & $38(25.0)$ & $53(32.7)$ & +39.5 \\
Overall transplant & 1,389 & 1,409 & +1.4 \\
Liver transplant & 515 & 543 & +5.4 \\
Living donor & $385(74.8)$ & $407(75.0)$ & +5.7 \\
Deceased donor & $130(25.2)$ & $136(25.0)$ & +4.4 \\
Kidney transplant & 757 & 774 & +2.2 \\
Living donor & $493(65.1)$ & $473(61.1)$ & -4.1 \\
Deceased donor & $264(34.9)$ & $301(38.9)$ & +14.0 \\
Heart transplant & 66 & 53 & -19.7 \\
Lung transplant & 51 & 39 & -23.5 \\
\hline
\end{tabular}

Values are presented as number (\%).

transplantation and deceased donor transplantation for our patients. To date, we have not had any instances of COVID-19 infection among our patients.

As the outbreak grew to a pandemic, many healthcare providers raised the concern that organ transplant recipients might be at higher risk of developing severe illness caused by COVID-19 [5]. In prior outbreaks of respiratory viruses, organ transplant recipients demonstrated greater susceptibility to infection, greater disease severity, and prolonged shedding of virus compared with the general population $[16,17]$. However, there are limited data on COVID-19 in organ transplant recipients [18]. Although organ transplant recipients might be more susceptible to COVID-19 infection, it is uncertain whether immunosuppression alters the clinical features and disease severity of COVID-19. According to data collected so far, the clinical features of COVID-19 among transplant recipients are as heterogeneous as they are in the general population [19]. Furthermore, there are some preliminary reports that the reduced immune response due to immunosuppression might have a protective effect against severe COVID-19 symptoms $[20,21]$.

Another concern for organ transplantation during the COVID-19 crisis is a lack of medical resources [22]. Healthcare providers are under increasing pressure to provide care only in the most urgent cases in order to preserve resources for the COVID-19 response [23]. A lack of ICU beds and ventilators might not only affect care for transplant recipients but also organ procurements from deceased donors [6]. In the face of unprecedented challenges posed by 
COVID-19, some decrease in transplant activities is inevitable $[7,24]$. Unfortunately, the pandemic will not disappear soon, and there is potential for a second wave of COVID-19 [25]. It is difficult to know when transplant activities will be able to return safely to previous levels. Therefore, transplant programs have to take measures to continue performing organ transplantations with appropriate infection control measures based on the local environment $[5,6]$. During this unprecedented crisis, performing organ transplants is a demanding work. We greatly appreciate the efforts of all medical staffs who are working tirelessly during this outbreak. Our experiences provide insights on how to recover after the peak of the pandemic. It is important to note that infection control measures should be modified accordingly as the situation continues to evolve.

Although transplantations are categorized as Tier $3 \mathrm{~b}$ ("do not postpone") in the Centers for Medicare and Medicaid Services recommendations for limiting nonessential elective surgeries, elective living donor transplantations have been postponed in transplant centers around the world $[6,7,24]$. Although living donor transplantations might be on an "elective" schedule, they cannot be considered elective for many patients with end-stage organ disease. The need for a living donor liver transplant can be life-threatening if it is not met within a reasonable time frame. For patients in need of a kidney transplant, it is unsafe to delay living donor transplantation because of the need for those patients to regularly visit dialysis facilities, where they may not be able to practice safe social distancing [26]. Because it is unclear how long the COVID-19 pandemic will continue, postponement of living donor transplantation might be devastating for patients with end-stage organ disease. Therefore, healthcare providers should carefully weigh the risks and benefits of postponing living donor transplantation, even under the dire circumstances of COVID-19.

Although the impact of COVID-19 is resonating across the world, many aspects of COVID-19 remain unknown. For example, we still do not know the true prevalence, transmission rate, and fatality rate [27]. In addition, we do not know the risk of donor-derived transmission of COVID-19, nor do we fully understand the immune response to COVID-19 or how long the immunity lasts [28]. Facing up to the uncertainties of COVID-19, best effort to overcome these uncertainties is massive test $[29,30]$. We acknowledge the limited accuracy of real-time RT-PCR and the risks of false negative results. In addition, widespread real-time RT-PCR testing will produce many true-negative results and might therefore seem to be wasteful during times of constrained resources [29]. Despite those caveats, extensive testing can reduce nosocomial transmission and protect healthcare providers as well as vulnerable patients from COVID-19. In our experience, ambitious testing has allowed us to maintain the very best care for patients with end-stage organ disease during the COVID-19 outbreak.

Our study has some limitations. First, it is mostly based on a small number of patients from a single center, although we did investigate the overall transplant activities in South Korea. Second, no patients with COVID-19 have undergone transplantation in our institution.

The COVID-19 pandemic has impacted healthcare systems around the globe. In the face of the COVID-19 pandemic, we acknowledge the necessity of careful resource allocation. In our experience, the maintenance of transplant activities during the COVID-19 pandemic has required more efforts and resources than what were required in the past for the same activities. Despite the heightened resource consumption due to the COVID-19 pandemic, we must continue to try to provide the best care for our patients. In the absence of a vaccine or effective treatment, stringent infection control measures and continued collaboration among transplant societies are required to get through the crisis. A systemic support from government is also needed to sustain lifesaving organ transplants programs. Our early experiences suggest that organ transplantation is feasible under appropriate infection prevention measures. At this time, appropriate infection prevention measures and extensive testing are essential strategies for the maintenance of transplant activities for our patients.

\section{ACKNOWLEDGMENTS}

\section{Conflict of Interest}

No potential conflict of interest relevant to this article was reported.

\section{ORCID}

Juhan Lee

Eun Jin Kim

Kyong Ihn

Jae Geun Lee

Dong Jin Joo https://orcid.org/0000-0003-4910-2596 https://orcid.org/0000-0002-5438-0649 https://orcid.org/0000-0002-6161-0078 https://orcid.org/0000-0002-6722-0257 https://orcid.org/0000-0001-8405-1531 
Myoung Soo Kim https://orcid.org/0000-0002-8975-8381

Soon II Kim https://orcid.org/0000-0002-0783-7538

Yu Seun Kim https://orcid.org/0000-0002-5105-1567

Kyu Ha Huh

\section{Author Contributions}

Conceptualization: JL, KHH. Data curation: JL. Formal analysis: JL, EJK, KI, JGL, DJJ. Investigation: JL, MSK, SIK, YSK, KHH. Methodology: JL, EJK. Project administration: JL, KHH. Supervision: MSK, SIK, YSK, KHH. Visualization: JL. Writing-original draft preparation: JL. Writing-review \& editing: JL, $\mathrm{KHH}$.

\section{REFERENCES}

1. World Health Organization. WHO coronavirus disease (COVID-19) dashboard [Internet]. Geneva: World Health Organization [cited 2020 May 18]. Available from: https://covid19.who.int/.

2. Zhou F, Yu T, Du R, Fan G, Liu Y, Liu Z, et al. Clinical course and risk factors for mortality of adult inpatients with COVID-19 in Wuhan, China: a retrospective cohort study. Lancet 2020;395:1054-62.

3. Gori A, Dondossola D, Antonelli B, Mangioni D, Alagna L, Reggiani P, et al. Coronavirus disease 2019 and transplantation: a view from the inside. Am J Transplant 2020;20:1939-40.

4. Michaels MG, La Hoz RM, Danziger-Isakov L, Blumberg EA, Kumar D, Green M, et al. Coronavirus disease 2019: implications of emerging infections for transplantation. Am J Transplant 2020;20:1768-72.

5. Kumar D, Manuel O, Natori Y, Egawa H, Grossi P, Han SH, et al. COVID-19: a global transplant perspective on successfully navigating a pandemic. Am J Transplant 2020;20:1773-9.

6. Angelico R, Trapani S, Manzia TM, Lombardini L, Tisone G, Cardillo M. The COVID-19 outbreak in Italy: initial implications for organ transplantation programs. Am J Transplant 2020;20:1780-4.

7. Boyarsky BJ, Po-Yu Chiang T, Werbel WA, Durand CM, Avery RK, Getsin SN, et al. Early impact of COVID-19 on transplant center practices and policies in the United States. Am J Transplant 2020;20:1809-18.

8. Korean Network for Organ Sharing. Current status for organs transplantation [Internet]. Cheongju: Korean Network for Organ Sharing [cited 2020 May 15] Avail-

able from: https://www.konos.go.kr/.

9. Lee J, Huh KH. Kidney transplantation trends in South Korea during the COVID-19 pandemic. Kidney Int 2020;98:512-3.

10. Korean Society for Transplantation. Guidance on coronavirus disease 2019 (COVID-19) for transplant clinicians [Internet]. Seoul: Korean Society for Transplantation; 2020 [cited 2020 March 21]. Available from: https://www.mykst.org.

11. Lee J, Lee JG, Kim S, Song SH, Kim BS, Kim HO, et al. The effect of rituximab dose on infectious complications in $\mathrm{ABO}$-incompatible kidney transplantation. Nephrol Dial Transplant 2016;31:1013-21.

12. Lee J, Lee JG, Lee JJ, Kim MS, Ju MK, Choi GH, et al. Results of $\mathrm{ABO}$-incompatible liver transplantation using a simplified protocol at a single institution. Transplant Proc 2015;47:723-6.

13. COVID-19 National Emergency Response Center; Epidemiology \& Case Management Team; Korea Centers for Disease Control \& Prevention. Contact transmission of COVID-19 in South Korea: novel investigation techniques for tracing contacts. Osong Public Health Res Perspect 2020;11:60-3.

14. Song JY, Yun JG, Noh JY, Cheong HJ, Kim WJ. Covid-19 in South Korea: challenges of subclinical manifestations. N Engl J Med 2020;382:1858-9.

15. Park S, Choi GJ, Ko H. Information technology-based tracing strategy in response to COVID-19 in South Korea-privacy controversies. JAMA 2020;323:2129-30.

16. Fishman JA, Grossi PA. Novel Coronavirus-19 (COVID-19) in the immunocompromised transplant recipient: \#Flatteningthecurve. Am J Transplant 2020;20:1765-7.

17. Kumar D, Tellier R, Draker R, Levy G, Humar A. Severe Acute Respiratory Syndrome (SARS) in a liver transplant recipient and guidelines for donor SARS screening. Am J Transplant 2003;3:977-81.

18. Zhong Z, Zhang Q, Xia H, Wang A, Liang W, Zhou W, et al. Clinical characteristics and immunosuppressant management of coronavirus disease 2019 in solid organ transplant recipients. Am J Transplant 2020;20:1916-21.

19. Columbia University Kidney Transplant Program. Early description of coronavirus 2019 disease in kidney transplant recipients in New York. J Am Soc Nephrol 2020;31:1150-6.

20. Bhoori S, Rossi RE, Citterio D, Mazzaferro V. COVID-19 in long-term liver transplant patients: preliminary experience from an Italian transplant centre in Lombardy. 
Lancet Gastroenterol Hepatol 2020;5:532-3.

21. D'Antiga L. Coronaviruses and immunosuppressed patients: the facts during the third epidemic. Liver Transpl 2020;26:832-4.

22. Emanuel EJ, Persad G, Upshur R, Thome B, Parker $M$, Glickman A, et al. Fair allocation of scarce medical resources in the time of COVID-19. N Engl J Med 2020;382:2049-55.

23. Elizabeth Brindle M, Gawande A. Managing COVID-19 in surgical systems. Ann Surg 2020;272:e1-2.

24. Tzedakis S, Jeddou H, Houssel-Debry P, Sulpice L, Boudjema K. COVID-19: thoughts and comments from a tertiary liver transplant center in France. Am J Transplant 2020;20:1952-53.

25. Leung K, Wu JT, Liu D, Leung GM. First-wave COVID-19 transmissibility and severity in China outside Hubei after control measures, and second-wave scenario planning: a modelling impact assessment. Lancet 2020;395:1382-93.

26. Ikizler TA. COVID-19 and dialysis units: what do we know now and what should we do? Am J Kidney Dis 2020;76:1-3.

27. Rajgor DD, Lee MH, Archuleta S, Bagdasarian N, Quek SC. The many estimates of the COVID-19 case fatality rate. Lancet Infect Dis 2020;20:776-7.

28. Cao X. COVID-19: immunopathology and its implications for therapy. Nat Rev Immunol 2020;20:269-70.

29. Lipsitch M, Swerdlow DL, Finelli L. Defining the epidemiology of COVID-19: studies needed. N Engl J Med 2020;382:1194-6.

30. Arons MM, Hatfield KM, Reddy SC, Kimball A, James A, Jacobs JR, et al. Presymptomatic SARS-CoV-2 infections and transmission in a skilled nursing facility. $\mathrm{N}$ Engl J Med 2020;382:2081-90. 